\title{
Algunas reflexiones sobre apnea obstructiva del sueño, ansiedad y consumo de alcohol
}

\section{Reflections about Obstructive Sleep Apnea, Anxiety, and Alcohol} Consumption

Recibido: 09 Septiembre 2020 | Aceptado: 24 Noviembre 2020

\author{
Carlos Mejía Avellaneda ${ }^{a}$ \\ Estudiante de la Especialización en Medicina Familiar, Facultad de Medicina, \\ Pontificia Universidad Javeriana, Bogotá, Colombia \\ ORCID: https://orcid.org/0000-0002-3097-7723 \\ José Antonio Garciandía Imaz \\ Profesor del Departamento de Medicina Preventiva y Social, Facultad de Medicina, \\ Pontificia Universidad Javeriana, Bogotá, Colombia \\ ORCID: https://orcid.org/0000-0002-3409-5998
}

a Autor de correspondencia: carlos_mejia@javeriana.edu.co

Cómo citar: Mejía Avellaneda C, Garciandía Imaz JA. Algunas reflexiones sobre apnea obstructiva del sueño, ansiedad y consumo de alcohol. Univ. Med. 2021;62(1). https://doi.org/10.11144/Javeriana.umed 62-1.aods

\section{RESUMEN}

Introducción: La apnea obstructiva del sueño (AOS) es un síndrome prevalente, caracterizado por episodios repetidos de obstrucción completa o parcial de las vías respiratorias superiores, en el cual ocurre un colapso faríngeo repetitivo durante el sueño, produce una respiración interrumpida durante la noche (hipoxia nocturna), fragmentación del sueño y excitación simpática. Puede acompañarse de algunas alteraciones en señales bioquímicas con subsecuentes manifestaciones de ansiedad, desbalance en patrones de apetito y saciedad o persistencia de etilismo. Objetivo: Reflexionar sobre las posibles conexiones de la AOS y el consumo abusivo de alcohol. Metodología: Revisión narrativa de la literatura médica publicada en inglés y español en bibliotecas de bases de datos biomédicas. La búsqueda se orientó con los términos apnea obstructiva de sueño, niveles de cortisol, grelina y leptina, alcoholismo. Discusión:Se encontró información de concentraciones alteradas en las hormonas cortisol, grelina y leptina, subsecuentes a las alteraciones generadas por la AOS en sus respectivos ciclos. Ello permite pensar en algún tipo de indicio respecto al consumo de alcohol por vía de la ansiedad (cortisol), la voracidad (grelina) y la saciedad (leptina). Conclusiones: la AOS modifica los ciclos hormonales de cortisol, grelina y leptina, que se manifiestan en el estado de vigilia con ansiedad, voracidad y dificultades de saciedad, tres síntomas que se presentan en el consumidor de altos niveles de alcohol. Esta coincidencia no se ha estudiado lo suficiente.

\section{Palabras clave}

alcoholismo; apnea; cortisol; leptina; grelina.

\begin{abstract}
Introduction: Obstructive sleep apnea (OSA) is a prevalent syndrome, characterized by repeated episodes of complete or partial obstruction of the upper airways, in which a repetitive pharyngeal collapse occurs during sleep, resulting in interrupted breathing at night (nocturnal hypoxia), sleep fragmentation, and sympathetic arousal. It may be accompanied by some alterations in biochemical signals with subsequent manifestations of anxiety, imbalance in patterns of appetite and satiety, persistence of alcoholism. Objective: To propose an explanatory hypothesis on the influence of OAS as a factor or influential element on the origin and
\end{abstract}


persistence of abusive alcohol consumption. Methodology: Systematic revision of medical literature published in English and Spanish in biomedical databases, related to the biochemical consequences of OSA in the levels of cortisol, ghrelin and leptin. Discussion: Information is found about the alterations of cortisol, ghrelin and leptin because of OSA, which allows to think in a relationship between apnea as a risk factor for alcohol consumption. Conclusions:Sleep disorders generate alterations in the cycle of cortisol, leading to an increase of its levels. Chronic reduction of sleep increases the activity of the sympathetic nervous system which translates into anxiety. Persons with moderate and severe OAS have higher appetite during night due to the increase of cortisol levels at that time of the day. Ghrelin is increased in persons with OSA, which translates into an increase in appetite and ingestion avidity. Leptin and OSA are closely related in affecting sleep architecture.

Keywords

alcoholism; apnea; cortisol; leptin; ghrelin.

\section{Introducción}

Sabemos, por la literatura médica, que el consumo de alcohol (abuso y alcoholismo) agrava los síntomas de la apnea obstructiva de sueño (AOS), que se manifiestan en un aumento del índice de apneas e hipopneas. Sin embargo, la literatura no muestra la influencia de la AOS en el origen, mantenimiento y expresiones del consumo anormal de alcohol y su expresión más grave: el alcoholismo. Uno de los autores, en su consulta habitual, recogió una muestra de 11 casos de alcoholismo en personas entre los 26 y los 38 años, en quienes después de practicárseles un polisomnograma basal se comprobó que presentaban un índice de apnea obstructiva, bien fuera moderada (un promedio de 25 ) o bien fuera severa (un promedio de 40).

Ante este hecho, a cada paciente se le realizó la historia clínica con la presencia de la madre, a quien se interrogó sobre los trastornos del sueño del paciente. En todos los casos se comprobó por la historia familiar que habían comenzado con los problemas de sueño en la infancia o en la adolescencia. Curiosamente, de los 11 casos, después de consultar con el otorrinolaringólogo, 10 presentaron desviación del tabique nasal. Todos ellos comenzaron con el consumo abusivo de alcohol entre los 16 y los 22 años, derivado posteriormente con los años en alcoholismo. Esta circunstancia nos indujo a los autores a hacernos una pregunta: si el consumo de alcohol agrava, como ya lo sabemos, el cuadro clínico de la AOS, ipodría plantearse lo contrario?, es decir ila AOS puede ser un factor de riesgo para el inicio del consumo de alcohol de forma abusiva y su expresión en alcoholismo? Con esta premisa iniciamos esta revisión narrativa en la literatura médica.

La AOS es un síndrome caracterizado por episodios repetidos de obstrucción completa o parcial de las vías respiratorias superiores, cursa con el colapso faríngeo repetitivo durante el sueño y produce respiración interrumpida durante la noche (hipoxia nocturna), fragmentación del sueño y excitación simpática (1). Las alteraciones fisiopatológicas, metabólicas y la carga alostática que puede suscitar se traducen en alteración en los pulsos de ciertas vías reguladoras que incluyen el apetito, la saciedad y la ansiedad. Con base en estos hallazgos, se buscó reflexionar sobre el papel que pueden tener la grelina (hambre), la leptina (saciedad) y el cortisol (ansiedad) en el estado fisiológico de un individuo, y si su alteración distorsiona lo suficiente como para propiciar una posible relación anormal con el consumo de alcohol (2).

La prevalencia de AOS en los Estados Unidos se ha estimado en alrededor del $15 \%$ en hombres y en un $5 \%$ en mujeres para personas de 30 a 70 años. La AOS está presente en el $41 \%$ de los pacientes con índice de masa corporal superior a 28 (3). También es común en adultos mayores, fumadores, en enfermedad arterial coronaria, derrame cerebral, hipertensión arterial y diabetes mellitus $(4,5)$. El AOS es una patología común en quienes son dependientes del alcohol, pues este aumenta el colapso de las vías respiratorias superiores y contribuye a un mayor índice de masa corporal (6).

En las últimas dos décadas, la prevalencia ha aumentado a un $10 \%$ en hombres de 30 a 49 an\# os, en un $17 \%$ en hombres de 50 a 70 , en un $3 \%$ en mujeres de 30 a 49 y en un $9 \%$ en mujeres de 50 a $70(7,8,9)$. La mayoría de los adultos con AOS moderado y severo no están diagnosticados. En los Estados Unidos, los estudios muestran 
que este síndrome afecta del $4 \%$ al $24 \%$ de los hombres y del $2 \%$ al $9 \%$ de las mujeres, al punto que se ha estimado que un $20 \%$ de los adultos de edad media tienen AOS leve y un $80 \%$ de los casos permanecen sin diagnosticar (10).

En Colombia, la prevalencia de los trastornos del sueño fue del $27 \%$, y constituye un problema de salud pública. Sin embargo, en el país no se cuenta con datos exactos de la frecuencia de la enfermedad. En un estudio realizado en 2016 se muestra la prevalencia global de alto riesgo de apnea del sueño en Bogotá Bucaramanga y Santa Marta, con el Cuestionario Berlín, del 19 \% (IC95 \%: 17,3-20,8 \%), y con la Escala STOPBANG, del 26,9\% (IC95\%: 24,9-29,0\%). En Cali, en el Centro Médico ONIRIS, la escala de Epworth evaluó a 309 pacientes con diagnóstico de hipertensión arterial y se encontró un $67 \%$ de frecuencia de AOS con prevalencia mayor en hombres (11). Estos datos refuerzan la necesidad de evaluar la relación de este síndrome con otras alteraciones.

\section{Metodología}

En la figura 1 se muestra cómo se recopiló la información.

\section{Figura 1}

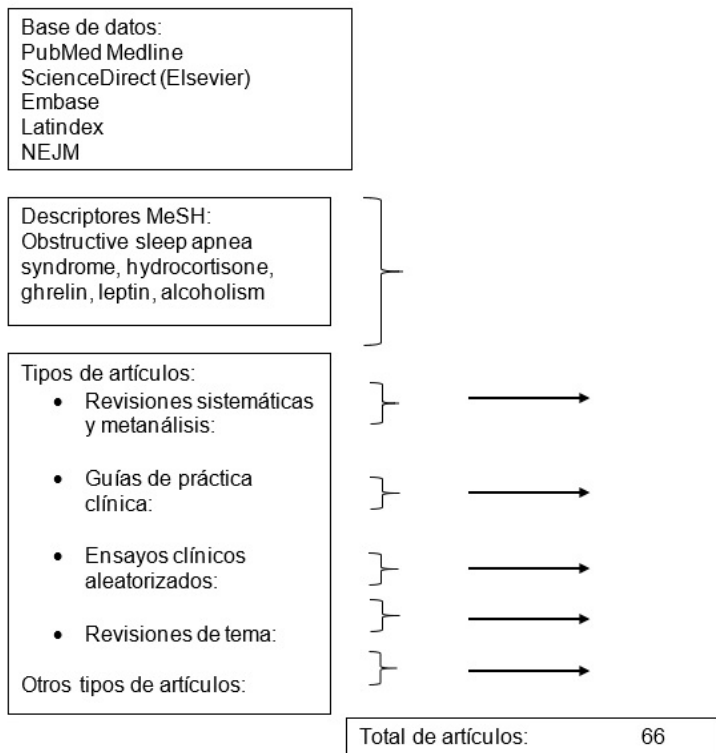

Síndrome de apnea obstructiva del sueño y alteraciones hormonales (cortisol, grelina y leptina)

La obstrucción de la vía aérea superior intermitente y la consiguiente hipoxia generadas por el AOS pueden conducir a una activación autonómica y provocar un despertar transitorio en repetidas ocasiones. La fragmentación del sueño derivada provoca somnolencia diurna, ansiedad, pobre desempeño laboral y mayor riesgo de accidentes automovilísticos y ocupacionales. La importancia clínica de la apnea se manifiesta en el hallazgo de un vínculo causal, independiente de la obesidad, en el desarrollo de hipertensión y enfermedad cardiovascular $(1,2)$.

Los despertares nocturnos en la apnea están asociados con alteraciones en la actividad hipotalámo-pituitaria-suprarrenal (HPA), específicamente en un aumento de la liberación pulsátil de cortisol, producto glucocorticoide humano del eje HPA, cuyas funciones incluyen regulación metabólica (gluconeogénesis), presión sanguínea y supresión inmunológica. Las fluctuaciones a lo largo de la noche se relacionan con la homeostasis del sueño y su desregulación en los trastornos del sueño manifiesta algunos efectos fisiológicos $(3,4)$.

Los pacientes con AOS se dividen en tres categorías de gravedad según el índice de apneahipopnea (IAH). Un IAH de 5 a 14,9 eventos es leve; de 15 a 29,9 eventos es moderado, y más de 30 eventos por hora caracteriza un AOS severo $(5,6)$. Los pacientes con AOS moderada y grave presentan anomalías cardiometabólicas, concentraciones alteradas de cortisol, ácidos grasos y glucosa. Los elevados ácidos grasos explican la mayor prevalencia de diabetes, dislipidemia, enfermedad del hígado graso y disfunción endotelial en pacientes con AOS $(7,8)$. En adultos mayores con AOS se observa una hipercortisolemia durante el sueño, lo cual para algunos autores, se relaciona con el deterioro cognitivo $(9,10)$. 
Síndrome de apnea obstructiva del sueño y cortisol

La secreción de cortisol la controla el sistema nervioso central, por medio de las neuronas del núcleo paraventricular del hipotálamo, que sintetizan la hormona liberadora de corticotropina (CRH) y la secretan al sistema portal hipofisario. La CRH estimula las neuronas del lóbulo anterior de la hipófisis, donde sintetiza y secreta la hormona adrenocorticótropa (ACTH), la melanotropina (MSH), la hormona lipotrófica y la betaendorfina, con la mediación de un péptido de 241 aminoácidos, llamado proopiomelanotocortina. La ACTH ejerce su acción sobre la corteza suprarrenal estimulando la esteroidoge\#nesis $(11,12)$. La regulación de la secreción de la ACTH depende de múltiples estímulos y del factor hipotalámico estimulante de la corticotropina y de la corticoliberina (CRH). La secreción de la ACTH depende del ritmo circadiano, regulado por ciclos de luzoscuridad; además, su concentración está en su punto más bajo en torno a la medianoche, aumenta progresivamente hasta alcanzar un pico en la mañana, propiciado por la necesidad de generar fuentes de energía (glucosa) después de largas horas de sueño $(13,14)$. A lo largo del día, las concentraciones de cortisol disminuyen de manera lenta hasta el anochecer. El ciclo sistémico del cortisol estimula las células de la corteza suprarrenal para liberar glucocorticoides (cortisol, cortisona y corticosterona) a la circulación sistémica. Este proceso de biosíntesis de cortisol tiene lugar en la capa fasciculada de la corteza suprarrenal con una producción diaria de $10-20 \mathrm{mg}$. La vida media del cortisol es de 90 min y su concentración en el plasma es de 10 a $20 \mu \mathrm{g} / \mathrm{dl}$. Este proceso necesita un paso previo, de conversión de colesterol a pregnenolona, reacción catalizada por la enzima citocromo P450SCC de localización mitocondrial $(15,16)$.

Por vía sistémica, el cortisol alcanza el hipotálamo y la hipófisis, donde regulación negativa inhibe la secreción de la CRH y la ACTH para mantenerlos en cantidades adecuadas. El cortisol, molécula lipídica, requiere transcortina, una globina transportadora para su paso al torrente sanguíneo, a la que se une en un $80 \%$; mientras que el $15 \%$ utilizará la albúmina como trasportador y el $5 \%$ restante circula libre. Se unirá a la albúmina si su concentración supera el límite de 0,2-0,5 $\mu \mathrm{g} / \mathrm{dl}$. La transcortina aumenta con los estrógenos, en la hepatitis o en el embarazo, y disminuye en la cirrosis, la nefrosis, el hipertiroidismo y su función no es esencial para las acciones del cortisol. Este último, unido a proteínas, funciona como reservorio circulante que mantiene la entrega de cortisol a los diferentes tejidos $(17,18)$.

Las acciones biológicas del cortisol son múltiples, pues afectan virtualmente todo el organismo y varios mecanismos homeostáticos. Es liberado en respuesta al estrés y actúa para restablecer la homeostasis. Sin embargo, la secreción prolongada de cortisol, debida al estrés crónico o a una secreción excesiva observada en el síndrome de Cushing, da lugar a importantes cambios fisiológicos, como la obesidad, la hipertensión arterial y la intolerancia a la glucosa $(19,20)$. Las principales acciones son contrarrestar la insulina, contribuir a la hiperglucemia (al estimular la gluconeogénesis hepática) e inhibir la utilización periférica de la glucosa. Tiene influencia inotrópica positiva en el corazón, al aumentar el índice de trabajo del ventrículo izquierdo, un efecto permisivo de las acciones de la adrenalina y la noradrenalina, tanto en el corazón como en los vasos sanguíneos. En ausencia de glucocorticoides, puede producirse una disminución del gasto cardiaco y shock; en los estados de exceso de glucocorticoides con frecuencia se observa hipertensión $(21,22)$.

El cortisol tiene una función esencial en la regulación inmunológica, mediante la inhibición de la síntesis de glucolípidos y precursores de prostaglandinas junto a las acciones de la bradicinina. Bloquea la secreción y los efectos de la histamina y de las citocinas inflamatorias (factor de necrosis tumoral $\alpha$, IL-1 e IL-6). Estas acciones reducen la respuesta inflamatoria. Las dosis altas de glucocorticoides disminuyen los monocitos, los eosinófilos y los linfocitos, en especial las células T. Atraviesa con facilidad la barrera hematoencefálica y tiene efectos 
directos sobre el metabolismo cerebral. Estimula el apetito, produce insomnio y altera la memoria y la capacidad de concentración. El exceso leve o moderado de cortisol durante un período limitado genera euforia y bienestar; sin embargo, el de\#ficit de glucocorticoides se asocia con depresión clínica $(23,24)$.

El efecto del trastorno respiratorio asociado al sueño en el eje HPA se ha controvertido en estudios en humanos (25). La mayoría de los estudios son limitados, dado que el cortisol se midió con una sola muestra, y es importante una correlación de 24 horas de la medición. Las interacciones entre el eje HPA y la regulación del sueño son reconocidas: el estrés repetido inducido por la hipoxia intermitente crónica o la fragmentación del sueño, que alteran la secreción circadiana de cortisol (26). La apnea del sueño, a través de la hipoxia nocturna y las excitaciones electroencefalográficas repetitivas, conduce a una mayor secreción de cortisol, que a su vez puede contribuir y acelerar el empeoramiento de la obesidad visceral y el síndrome metabólico. Dentro de los estudios que abordan el tema, el publicado por Edwards et al. (17), en 2014, propone evaluar los cambios en la función de secreción nocturna del cortisol en la función cognitiva en pacientes con AOS grave que no habían recibido ningún tratamiento con presión positiva continua en las vías respiratorias (CPAP) antes del inicio del estudio. Entre tanto, el estrés hipóxico intermitente eleva la secreción de cortisol. Los despertares nocturnos experimentales causan una liberación significativa de cortisol pulsátil, correlacionado con lo observado en pacientes con insomnio crónico que muestran niveles elevados de cortisol, especialmente durante la noche y el periodo temprano de sueño nocturno. La falta de sueño a corto plazo aumenta el cortisol plasmático durante la tarde del día siguiente. En el insomnio crónico, el cortisol en el plasma y las concentraciones de ACTH están aumentados en la parte temprana de la noche. La reducción crónica del sueño se ha relacionado con cantidades elevadas de cortisol salival, el aumento de la actividad del sistema nervioso simpático y el deterioro de la tolerancia a la glucosa $(27,28)$.

\section{Cortisol y ansiedad}

Un estudio realizado por Panaree et al. (26) analizó muestras repetidas, con el fin de observar la evolución de la curva del cortisol durante el día según los niveles de depresión, ansiedad y reactividad emocional. Un subgrupo de adolescentes que mostraron concentraciones elevadas de cortisol por la noche presentaron mayor cantidad de síntomas psiquiátricos. En el mismo estudio se muestra la asociación entre los marcadores biológicos neuroendocrinos del estrés como el cortisol, el temperamento y los trastornos afectivos. En este contexto, la alteración del cortisol es generada por una activación de eje HPA como respuesta endocrina del organismo a amenazas reales o percibidas, psicológicas y fisiológicas. La respuesta al estrés es adaptativa; pero una activación prolongada del eje HPA, reflejada en niveles elevados de glucocorticoides, puede afectar negativamente diversos mecanismos biológicos y actuar como factor de riesgo para trastornos emocionales.

La exposición prolongada al cortisol puede conducir a cambios estructurales en las regiones del cerebro responsables de modular la respuesta al estrés y participar en la fisiopatología de la ansiedad y los trastornos del estado de ánimo (27). Así mismo, el estado de ánimo y el trastorno depresivo mayor se asocian con las respuestas al estrés del eje HPA. Aunque los estudios han evaluado las respuestas de cortisol en el contexto de la depresión clínica, pocos han investigado las asociaciones entre las respuestas de cortisol y la sintomatología depresiva subclínica. La asociación entre los síntomas depresivos y las respuestas de cortisol se muestra ambigua (28).

\section{Grelina y síndrome de apnea obstructiva del sueño}

La grelina es un péptido de 28 aminoácidos sintetizado principalmente por las células endocrinas del estómago, ubicadas en el fondo gástrico, en el intestino delgado, en el páncreas, 
en la hipófisis, en el riñón y en la placenta. Se secreta una o dos horas antes de comer y su concentración alcanza el pico, antes de comer, para disminuir una hora después, lo cual muestra su papel en la percepción del hambre. El paralelismo entre la percepción del hambre y la concentración de grelina es mayor prepandialmente que posprandialmente, lo que muestra que la grelina es un iniciador de la acción de comer, pero no un factor que haga que esta finalice. Tal disminución en la concentración de grelina plasmática posprandial puede estar relacionada con la composición de los alimentos ingeridos, dado que la inhibición de su secreción está relacionada con la hiperglucemia $(29,30)$.

La grelina regula el apetito de forma directa, activando las neuronas sensibles al neuropéptido Y (NPY) y al péptido relacionado agouti (AgRP), neuropéptido producido en el cerebro por la neurona AgRP/NPY. Se sintetiza en la región ventromedial del núcleo arqueado del hipotálamo. Potente péptido orexigénico, a su vez, modula el balance para la leptina y la insulina. Así mismo, activa las fibras nerviosas aferentes que inervan el núcleo del tracto solitario que, a su vez, envía señales a los núcleos hipotalámicos reguladores del hambre y de la energía. Estimula la liberación de cortisol, el cual disminuye la producción de citocinas (inhibe su expresión en las células T activadas), disminuyendo las concentraciones de citocinas proinflamatorias anorexigénicas: IL-1 $\beta$, IL-6, IFN- $\alpha$, IFN- $\gamma$ y TNF- $\alpha(31,32)$.

La grelina antagoniza a la leptina por activación de la vía del receptor hipotalámico del neuropéptido Y, y su administración estimula la secreción de hormona de crecimiento, produciendo un aumento de peso y adiposidad, al incrementar la ingestión de alimentos y reducir la utilización de grasa. Por otra parte, la leptina sintetizada a partir del tejido adiposo disminuye la ingestión de alimentos y aumenta el gasto energético. El efecto de la grelina en el gasto energético es controvertido, aunque muestra una disminución dosis dependiente en el consumo de oxígeno y en la temperatura corporal. La grelina probablemente también estimula la adipogénesis, al reducir la utilización de grasa en ratas e incluso puede inhibir la lipólisis (32). En la privación total de sueño o el sueño restringido, los participantes elevaron la grelina y redujeron la leptina, lo que produce una mayor predisposición a ganancia de peso a largo plazo $(30,31)$.

La grelina es una de las hormonas relacionadas con el abuso del alcohol; está involucrada en la regulación de la ingesta de alimentos y el equilibrio energético. Los hallazgos sugieren su participación en la modulación de las vías de recompensa mesolímbicas, por lo que es potencialmente relevante en la fisiopatología de los trastornos, por uso de sustancias como la dependencia del alcohol (33). La evidencia preclínica sugiere que la grelina está involucrada en la búsqueda de ingesta de alcohol, ya que modula las rutas centrales de recompensa y estrés. Sin embargo, no está claro cómo la administración de grelina puede afectar el consumo de alcohol en humanos. La grelina intravenosa, comparada con placebo, aumentó significativamente el número de infusiones de alcohol autoadministradas en murinos. Conocida como la "hormona del hambre", tiene un papel establecido en el aumento del apetito (34).

En humanos, la administración de grelina aumenta la necesidad de ingesta, provocada por señales en los bebedores fuertes y dependientes del alcohol $(35,36)$. Las concentraciones de acilgrelina en el plasma y el fondo gástrico en ayunas fueron más bajas en pacientes con dependencia del alcohol ingresados en el hospital por abstinencia de alcohol, en comparación con aquellos en los controles (37). Del mismo modo, las cantidades de grelina total en ayunas fueron más bajas en pacientes varones con dependencia del alcohol que consumieron al menos 80 gramos de alcohol durante las 24 horas anteriores en comparación con los controles. Otro estudio (38) informó que las concentraciones de grelina en ayunas son mayores en pacientes masculinos con dependencia al alcohol después de una abstinencia de al menos 30 días, en comparación con aquellos en los controles que no tenían antecedentes de dependencia del alcohol y se abstuvieron del alcohol durante al menos 72 horas. En el grupo de dependencia del alcohol, la grelina en ayunas aumentaba y se 
correlacionó positivamente con la duración de la abstinencia, y negativamente con la ingesta diaria de alcohol antes de la abstinencia. Los pacientes con dependencia del alcohol muestran una grelina total en ayunas más alta dentro de los 10 días posteriores a la hospitalización, en comparación con los controles. En otro estudio (39), los abstemios tempranos (que tomaron su último trago de 24 a 72 horas antes) muestran cantidades de grelina total en ayunas más altas que los bebedores activos. En el mismo estudio, en una muestra de 61 pacientes varones hospitalizados con dependencia del alcohol, se observó una correlación positiva entre las puntuaciones de acilgrelina en ayunas y ansia de alcohol, tanto al inicio de la abstinencia de alcohol como después de 14 días de abstinencia.

\section{Leptina y síndrome de apnea obstructiva del sueño}

La leptina es una hormona peptídica producida principalmente en el tejido adiposo, relacionada con la regulación de la homeostasis energética, inflamación, metabolismo y actividad nerviosa simpática. Las cantidades de leptina se correlacionan de manera positiva con las mediciones de adiposidad y con la fisiopatología de las enfermedades asociadas con la obesidad $(40,41)$.

Regula la función ventilatoria por medio del sistema nervioso central y el sistema nervioso periférico (mediante respuesta quimiorrefleja del cuerpo carotídeo). La hipoxia intermitente crónica aumenta la señalización de leptina en el cuerpo carotídeo y la inyección periférica de leptina aumenta el volumen respiratorio en respuesta a la hipoxemia (42). La administración de leptina en el lugar de quimiorrecepción central primaria en la médula mejora la respuesta ventilatoria a la hipercapnia en modelos animales. Dado su papel en la función cardiovascular y respiratoria, la leptina se investiga cada vez más para comprender la fisiopatología de las enfermedades asociadas con la obesidad $(34,43)$.

Investigadores en estudios experimentales y humanos han evaluado el papel de la leptina en la patogénesis de la AOS, y actualmente la mejor evidencia muestra que la leptina altera la arquitectura del sueño y provoca cambios en la función ventilatoria y la resistencia de las vías aéreas superiores. Muchos de estos hallazgos se derivan de experimentos en modelos animales con bajo nivel de leptina funcional, sin leptina o con receptores de leptina mutados que impiden que actúe sobre las células diana $(44,45)$.

Las concentraciones plasmáticas de leptina en humanos pueden no reflejar los efectos en el tejido central o periférico y, probablemente, indican un estado resistente a la leptina o "deficiencia funcional" de esta. Esto puede explicar los resultados discrepantes derivados de los estudios en animales versus humanos y el supuesto papel de la leptina como biomarcador del riesgo o pronóstico de enfermedad $(46,47)$.

Un estudio en humanos que utilizó mediciones únicas de leptina demostró que las cantidades de leptina en el plasma son elevadas entre las personas con AOS, en comparación con aquellos sin el síndrome (48). Existe una modesta correlación entre la leptina plasmática y el IAH, un indicador de la gravedad de la AOS. Otro estudio mostró que existe una correlación significativa entre las mediciones de adiposidad (por ejemplo, circunferencia de la cintura e índice de masa corporal) y los niveles de leptina (49). La elevación de las concentraciones de leptina en la AOS fue independiente del índice de masa muscular o la circunferencia de la cintura (49). Sin embargo, otro estudio señaló una asociación entre el IAH y la leptina, independientemente de las mediciones de adiposidad (50).

La terapia con CPAP reduce la leptina en unos pocos días; del mismo modo, el tratamiento quirúrgico efectivo de la AOS (moderada a grave) con úvulo-palato-faringoplastia reduce los niveles de leptina. Los efectos a largo plazo del tratamiento con CPAP muestran una disminución de sus concentraciones (51). En un estudio longitudinal, los cambios en la leptina después de 6 meses de tratamiento con CPAP en 96 pacientes con AOS muestran que tanto el índice de masa corporal reducido como el 
IAH son predictores independientes de leptina reducida (52).

Los pacientes con AOS grave tienen mayores concentraciones de leptina en el plasma que los pacientes con AOS leve. Existe una correlación positiva, independiente de la edad y el índice de masa corporal, entre la leptina en el plasma y la gravedad de AOS, lo que sugiere que la hiperleptinemia puede ser un marcador pronóstico de AOS (53).

La leptina afecta la arquitectura del sueño, la ventilación y la defensa de la permeabilidad de las vías aéreas superiores, aparte de que participa en la patogénesis de la AOS, pero los mecanismos por los cuales la leptina afecta la expresión del AOS no son claros (54).

\section{Alcoholismo}

El alcohol es la segunda sustancia psicotrópica de mayor uso en el mundo, un factor de riesgo de muerte y de discapacidad temprana. Presenta un alto grado de asociación con enfermedades mentales, traumatismos, enfermedad coronaria, cirrosis hepática, epilepsia y cáncer (hepático, colorrectal, mamario, laríngeo, faríngeo y esofágico). Tiene impacto en el grado de discapacidad y en el funcionamiento familiar, económico y social de los individuos (agresiones, situaciones de violencia, intentos suicidas, suicidios consumados, uso de otras sustancias psicoactivas) (55).

En el Estudio Nacional de Consumo de Sustancias Psicoactivas en Colombia (2013) se encontró que el $87 \%$ había consumido alcohol alguna vez en su vida, y de estos, el 35,8 \% en los últimos 30 días: un $91 \%$ para varones y un $83 \%$ para mujeres. El consumo riesgoso de alcohol se encuentra en los jóvenes de 18 a 24 años $(49,2$ $\%)$ y los de 25 a 34 años (45,4\%) (56).

La Organización Mundial de la Salud define como un consumo habitual de alcohol un valor de 20-40 gramos por día en las mujeres y 40-60 gramos por día en varones. Los sujetos que presentan este tipo de consumo pueden manifestar un deseo intenso de conseguir la bebida, incluso un fracaso en los intentos por controlar el consumo, pese a conocer su uso problemático (56).

Entre los factores de riesgo para sufrir un trastorno por consumo de alcohol figuran antecedentes personales de enfermedad mental, uso de otras sustancias psicoactivas, problemas durante la infancia, la dinámica familiar y modelos de crianza, experiencias emocionalmente perturbadoras y enfermedades no psiquiátricas crónicas (57).

En un estudio (58) se mostró la asociación entre la depresión y los trastornos del estado de ánimo con el consumo problemático de alcohol. Hay un riesgo de depresión entre dos y tres veces mayor si hay dependencia del alcohol, de modo que es una comorbilidad bidireccional. De los sujetos con antecedentes de trastornos de ansiedad, hasta el $12 \%$ tiene trastorno por consumo de alcohol y pueden empeorar los trastornos del sueño.

\section{Apnea del sueño y alcohol}

$\mathrm{El}$ alcohol es un relajante muscular que puede empeorar potencialmente la AOS. El impacto del alcohol en los parámetros respiratorios durante el sueño, según un metanálisis de catorce estudios (57), evidenció que el IAH aumentó significativamente después de la administración de alcohol y que la SO2 media se redujo significativamente. El aumento en el IAH fue mayor en los roncadores y aquellos con un diagnóstico de AOS. También se observa un aumento significativo de duración del evento respiratorio y una disminución en la $\mathrm{SO} 2$.

Un estudio (58) señaló que la AOS es más común entre las personas que consumen alcohol, posiblemente porque el alcohol aumenta la colapsabilidad de las vías respiratorias y porque contribuye a un mayor índice de masa corporal.

El alcohol aumenta el riesgo de AOS, reduce el tono muscular geniogloso, predispone a los pacientes al colapso de la vía aérea superior y, generalmente, aumenta la resistencia de la vía aérea superior (59). El consumo elevado de alcohol también contribuye a la ingesta calórica en la dieta y, por lo tanto, en algunos casos, a un 
índice de masa corporal alto, que en sí mismo es un factor de riesgo para la AOS, en consumidores altos, aumenta el riesgo de AOS en un $25 \%$ (60). Un estudio que presenta estimaciones ajustadas por el índice de masa corporal confirmó un efecto independiente del alcohol, donde la obesidad no es el mecanismo principal responsable del mayor riesgo de AOS entre quienes consumen alcohol y refiere que los trastornos del sueño son más comunes en relación con el consumo de alcohol (61).

La regularidad del consumo de alcohol es importante en su efecto sobre la AOS, ya que la relajación muscular de las vías respiratorias y la sensibilidad reducida a la apnea son probablemente mayores cuando aumentan las cantidades de alcohol en el organismo (62). El consumo de alcohol antes de acostarse y sus efectos sobre la arquitectura del sueño difieren en función de las concentraciones ascendentes o máximas durante las primeras 3-4 horas de la noche (primera mitad de la noche), en comparación con la fase descendente de los niveles de alcohol en sangre, durante la próximas 3-4 horas de sueño (segunda mitad de la noche). El efecto del alcohol moderado y abundante en el sueño en adultos sanos muestra una disminución de la duración del sueño de movimientos oculares rápidos (63).

\section{Discusión}

El consumo de alcohol y su influencia en el empeoramiento de AOS es un hecho ya descrito en la literatura médica; sin embargo, la relación inversa no ha merecido alguna reflexión. El presente artículo intentó explorar la posibilidad de algún indicio que partiendo de la presencia de AOS lleve al alcoholismo. Para ello, la revisión narrativa se centró en explorar los efectos del AOS sobre tres hormonas involucradas en el ciclo circadiano y el ciclo sueño-vigilia: cortisol, grelina y leptina. Concentrar la atención en ellas se ajusta a hechos que las alteraciones de estas generan en la clínica. La alteración del cortisol, si es en el sentido de un aumento, produce ansiedad; el aumento de la grelina genera un comportamiento voraz, ávido y compulsivo con la alimentación, y las alteraciones de la leptina, dificultades con el proceso de saciedad y un empeoramiento de la AOS. Bajo esta premisa, se buscó información sobre los efectos de la AOS en generar alteraciones en el sentido planteado. Los hallazgos en la literatura muestran diferentes aspectos que satisfacen la preocupación expresada.

En primer lugar, los despertares nocturnos experimentales causan un aumento significativo de cortisol pulsátil, similar a lo observado en pacientes con insomnio crónico, con predominio en el periodo temprano de sueño nocturno y continuando el resto de la noche (30). Los despertares nocturnos son característicos de la AOS, y el efecto de la apnea genera un aumento del cortisol (7). Las alteraciones que producen estrés hipóxico aumentan la secreción de cortisol. Así lo muestra una revisión (8) sobre las interacciones del eje HPA y el sueño, con el fin de evaluar la actividad normal de dicho eje y el ritmo circadiano con respecto a los trastornos del sueño. En el mismo estudio se expresa que pacientes con AOS grave y estrés hipóxico intermitente alteran la secreción de cortisol.

Estos aspectos muestran que las alteraciones del sueño (despertares, insomnio crónico e hipoxia), presentes en la AOS, aumentan la secreción de cortisol en diferentes momentos. Este aumento se traduce en expresiones clínicas del efecto del cortisol como ansiedad, angustia o inquietud, en ocasiones intensas. Los efectos ansiógenos del cortisol son propiciados por su facilidad para atravesar la barrera hematoencefálica, los efectos directos sobre el metabolismo cerebral, la estimulación del apetito, la producción de insomnio, la disminución de la memoria y la concentración que pueden desembocar en estados depresivos.

En este sentido, una persona con padecimiento de AOS, de moderada o severa intensidad, es muy posible que presente elevadas tasas de cortisol y que se le produzca un estado general ansioso-angustioso, acompañado de síntomas depresivos, que, junto con el aumento del apetito resultante, pueda inducir el consumo 
de alcohol como una forma de autotratamiento de esos síntomas mencionados. Con el tiempo, el consumo puede intensificarse y transformarse en un abuso de alcohol o alcoholismo. Sumado a ello, los hallazgos sugieren la participación del cortisol en la modulación de las vías de recompensa mesolímbicas (28), lo cual consolida el abuso y la dependencia del alcohol.

Por otra parte, el aumento de la grelina está involucrada en la búsqueda de ingesta de alcohol, ya que modula las rutas centrales de recompensa y estrés. Un estudio (29) mostró que la grelina intravenosa tuvo un aumento significativo en el número de infusiones de alcohol autoadministradas en múridos, comparado con el grupo placebo. La grelina está involucrada en el incremento del hambre en personas que padecen trastornos del sueño (AOS o insomnio), manifiesto en atracones nocturnos y la ingestión de alimentos de forma ansiosa. Un estudio (30) que examinó los efectos de la administración de grelina en ratones expuestos al alcohol durante nueve semanas, en el contexto de acceso limitado de libre elección de dos botellas, alcohol versus agua, la administración de acetil-grelina aumenta el consumo de alcohol. Y la administración periférica de un antagonista de la grelina conduce a una disminución del consumo de alcohol. En humanos, un estudio (31) halló que la administración de grelina aumenta el antojo inducido por señales, en bebedores fuertes y dependientes de alcohol. Según otro estudio (32), las concentraciones de acetil grelina en el fondo gástrico se muestran bajas en pacientes con dependencia de alcohol, ingresados por abstinencia de alcohol, en comparación con los controles, y ello prueba que aquellos quienes consumen alcohol con dependencia en curso tienen niveles más altos de grelina. Un estudio (37) señaló que la grelina total en ayunas es menor en pacientes varones con dependencia al alcohol que consumieron al menos 80 gramos de alcohol durante las 24 horas anteriores, en comparación con los controles. Otro estudio (38) informó que las cantidades de grelina en ayunas fueron más altas en pacientes masculinos con dependencia al alcohol, después de una abstinencia de, al menos, 30 días, en comparación de los controles que no tenían antecedente de dependencia de alcohol y se abstuvieron de consumo de alcohol en, al menos, 72 horas. En este punto, observamos que el aumento de cortisol y grelina como consecuencia de la AOS incrementan dos condiciones: ansiedad y voracidad, cuya naturaleza establece un contexto personal propicio para la búsqueda de calmarlas con la ingestión de alcohol.

En cuanto a la leptina, su aumento propicia episodios de obstrucción de las vías aéreas superiores e influye en el desequilibrio del metabolismo energético y la fisiopatología de la obesidad (33). También está involucrada en la regulación de la función ventilatoria central y periférica, que aumenta durante la hipoxia intermitente (39). Con relación a la AOS, altera la arquitectura del sueño, la función ventilatoria y la resistencia de la vía aérea superior, en modelos animales. En humanos se muestran concentraciones plasmáticas elevadas en comparación con los controles (40). Es importante anotar que las cantidades de leptina en humanos no reflejan los efectos de esta en los tejidos, lo cual dificulta su uso como biomarcador (41). A estos datos se suma una correlación entre mediciones de adiposidad y leptina, en los cuales los pacientes con AOS muestran una elevación la leptina, independiente del grado de adiposidad (42). Dentro de la respuesta terapéutica de la AOS a la terapia con CPAP se ha comprobado una reducción de las concentraciones de leptina y que el manejo quirúrgico efectivo ha generado la misma respuesta (43).

\section{Conclusión}

Con lo expuesto se observa que la AOS altera el ciclo del cortisol, expresado en un aumento de los pulsos de su secreción, en el atardecer y la noche, donde debe comenzar su descenso. El aumento del cortisol se manifiesta con ansiedad y angustia, además de síntomas distímicos y depresivos. Con ello las personas con AOS (moderado o severo) tienen más ansiedad, angustia y síntomas depresivos. La reducción crónica del sueño, como sucede en la AOS, estimula un aumento 
de la actividad del sistema nervioso simpático, que produce síntomas de ansiedad. El efecto ansiógeno del cortisol estimula el centro del apetito en el cerebro. Por ello, las personas con AOS (moderado o severo) tienen mayor apetito en las horas de la noche, efecto que se ve intensificado por el aumento también de producción de grelina en las personas con AOS, que suscita un incremento del apetito, la avidez por ingerir y la tendencia al consumo de alcohol. Así mismo, el cortisol, con su participación en la modulación de vías de recompensa mesolímbicas, contribuye en los procesos de abuso de sustancias y consolida el efecto de recompensa.

Por otra parte, la AOS cursa con una elevada secreción de leptina, que produce alteraciones ventilatorias de las vías superiores y en la arquitectura del sueño, estableciendo un círculo vicioso que se retroalimenta entre la AOS y la leptina, subsecuentemente agrava la AOS y contribuye de manera indirecta a un aumento de cortisol y grelina.

El conocimiento que se desprende de esta revisión sobre la AOS y su relación con la alteración de la secreción de cortisol, grelina y leptina nos muestra que las tres hormonas tienen su ciclo de secreción alterado hacia el alta. En consecuencia, suscitan en la persona que padece AOS (moderada o severa) un aumento de la ansiedad y la angustia, propiciadas por el incremento de cortisol y de la actividad del sistema nervioso simpático. Así mismo, el efecto del cortisol sobre el centro del apetito, junto con el aumento del apetito y de la avidez por ingerir que produce la grelina, predispone al consumo de sustancias que puedan calmar los síntomas. Una de esas sustancias que puede calmar transitoriamente la ansiedad, la angustia o la avidez oral es el alcohol. Unido a ello, el efecto reforzador del circuito de recompensa que el aumento de cortisol parece generar permite reflexionar en cuanto a que la AOS es capaz de contribuir a la generación del consumo de alcohol, su abuso y la cronicidad de su ingesta hasta llegar al alcoholismo. Dado que la leptina y la AOS mantienen un círculo vicioso de retroalimentación positiva que contribuye a agravarla, junto con el cortisol y la grelina, se puede pensar de forma razonablemente especulativa que:

La AOS, al aumentar la secreción de cortisol, grelina y leptina, contribuye a una cierta predisposición para el consumo de alcohol como una forma de calmar la ansiedad y la avidez que suscita.

Con este análisis conceptual, podemos extraer una conclusión de alcances muy limitados en cuanto al AOS y el consumo de alcohol. Sabida es la relación de la influencia del consumo elevado de alcohol en el empeoramiento de la AOS; sin embargo, en un sentido contrario (influencia de la AOS en el consumo de alcohol) solo encontramos indicios para una razonable reflexión especulativa que plantea la posibilidad de relacionar el efecto de la AOS en el consumo de alcohol. La pretensión de esta revisión tuvo el modesto propósito de la apertura de una línea de investigación para indagar respecto a la inquietud planteada.

\section{Referencias}

1. Tomfohr LM, Edwards KM, Dimsdale JE. Is obstructive sleep apnea associated with cortisol levels? A systematic review of the research evidence. Sleep Med Rev. 2012;16(3):243-9. http://dx.doi.org/10 $.1016 / j . s m r v .2011 .05 .003$

2. Chopra S, Rathore A, Younas H, Pham L V, et al. Obstructive sleep apnea dynamically increases nocturnal plasma free fatty acids, glucose, and cortisol during sleep. J Clin Endocrinol Metab. 2017 Sep;102:3172-81.

3. Crawford-Achour E, Saint Martin M, Roche F. Stress hormones in obstructive sleep apnea complications: the role of cortisol. Sleep Med. 2014;15(1):3-4. http://dx.doi.org/10.1 016/j.sleep.2013.10.004

4. Buckley TM, Schatzberg AF. Review: on the interactions of the hypothalamic-pituitaryadrenal (HPA) axis and sleep: 
Normal HPA axis activity and circadian rhythm, exemplary sleep disorders. J Clin Endocrinol Metab. 2005;90(5):3106-14.

5. Lattova Z, Keckeis M, MaurovichHorvat E, Wetter TC, Wildefrenz J, Schuld A, et al. The stress hormone system in various sleep disorders. J Psychiatr Res. 2011;45(9):1223-8. http://dx.doi.org/1 0.1016/j.jpsychires.2011.03.013

6. Basta M, Chrousos GP. Hypothalamic-pituitary-adrenal axis activity in obese men with and without sleep apnea: effects of continuous positive airway pressure therapy. J Clin Endocrinol Metab. 2014 Aug;92:4199-207.

7. Dadoun F, Darmon P, Achard V, Alessi MC, Rey M, Grino M, et al. Effect of sleep apnea syndrome on the circadian profile of cortisol in obese men. Am Physiol Soc. 2007;466-75.

8. Pinna Puissant S, Van Den Bergh BRH, Van Calster B, Van Huffel $S$ Relación del perfil del cortisol salival en adolescentes de 14-15 años de edad, con la depresión, ansiedad y reactividad emocional. Rev Mex Psicol [internet]. 2008;25(2):249-58. Disponible en: https://www.redalyc.org /articulo.oa? $\mathrm{id}=243016308005$

9. Fiksdal A, Hanlin L, Kuras Y, Gianferante D, Chen X, Thoma $\mathrm{MV}$, et al. Psychoneuroendocrinology associations between symptoms of depression and anxiety and cortisol responses to and recovery from acute stress. Psychoneuroendocrinology. 2019 Nov;102:44-52. https://doi.org/1 0.1016/j.psyneuen.2018.11.035

10. Potau Vilalta N; DPAC. Gonadotropinas (LH y $\mathrm{FSH}$ ) y corticotropina (ACTH). Endocrinol Nutr. 2007;54(2):109-17.
11. Gofferje M, Kern W, Born J, Fehm HL. Sleep disruption alters nocturnal cortisol secretory patterns. Soc Biol Psychiatry. 1991;29.

12. Hidalgo Martínez P, Lobelo R. Epidemiología mundial, latinoamericana y colombiana y mortalidad del síndrome de apneahipopnea obstructiva del sueño (SAHOS). Rev la Fac Med. 2017;65(1):40-3.

13. Ruiz ÁJ, Alonso M, Sepúlveda R, Hidalgo P, Cañón M, Otero L, et al. Prevalence of sleep complaints in Colombia at different. Sleep Sci. 2016;1-6. http://dx.doi.org/10.1016/j.sl sci.2016.05.008

\section{Cipriani E. Repercusiones endocrinólogicas en la apnea obstructiva del sueño. Rev Méd Hered. 2010;39-45.}

15. Londoño Palacio N, Rico-López AF. Repercusiones endocrinológicas del síndrome de apnea-hipopnea obstructiva del sueño (SAHOS). Rev Fac Med. 2017;65(suppl 1):S55-7.

16. Durán Agüero S, Fernández Godoy E, Fehrmann Rosas P, Delgado Sánchez C. Menos horas de sueño asociado con sobrepeso y obesidad en estudiantes de nutrición de una universidad chilena. Rev Peru Med Exp Salud Pública. 2016;33(2):264-8.

17. Edwards KM, Kamat R, Tomfohr LM, Ancoli-Israel S, Dimsdale JE. Obstructive sleep apnea and neurocognitive performance: the role of cortisol. Sleep Med. 2014;15(1):27-32. http://dx.doi.org/ $10.1016 /$ j.sleep.2013.08.789

18. Guerrero J. Para entender la acción de cortisol en inflamación aguda: una mirada desde la glándula suprarrenal hasta la célula blanco. Rev Med Chil. 2017;230-9. 
19. Fluerașu MI, Bocsan IC, Buduru S, Pop M, Vesa SC, Zaharia A, et al. The correlation between sleep bruxism, salivary cortisol, and psychological status in young, Caucasian healthy adults. J Craniomandib Sleep Pract. 2019;0(0):1-7. https://doi.org/10.1080/ 08869634.2019 .1619250

20. Wang E, Chomsky-higgins $\mathrm{K}$, Chen Y, Nwaogu I, Seib CD, Shen WT, et al. Treatment of primary aldosteronism reduces the probability of obstructive sleep apnea. J Surg Res. 2018;236:37-43. https://doi.org/10.101 6/j.jss.2018.10.040

21. Bozic J, Galic T, Supe-Domic $\mathrm{D}$, Ivkovic $\mathrm{N}$, Ticinovic Kurir $\mathrm{T}$, Valic Z, et al. Morning cortisol levels and glucose metabolism parameters in moderate and severe obstructive sleep apnea patients. Endocrine. 2016;53(3):730-9. https://doi.org/10.1 007/s12020-016-0925-6

22. Jeong J, Guilleminault C, Park C, Son H, Lee H, Hwang S, et al. Changes in salivary cortisol levels in pediatric patients with obstructive sleep apnea syndrome after adenotonsillectomy. Sleep Med. 2014. http://dx.doi.org/10. 1016/j.sleep.2013.12.019

23. Park C, Guilleminault C, Hwang S, Jeong J, Park D, Maeng J. Correlation of salivary cortisol level with obstructive sleep apnea syndrome in pediatric subjects. Sleep Med. 2013;14(10):978-84. http://dx.doi.org/ 10.1016/j.sleep.2013.05.012

24. Ghiciuc CM, Corina L, Cozma D, Bercea RM, Elena C, Mihaescu $\mathrm{T}$, et al. Restoring the salivary cortisol awakening response through. Chronobiol Int. 2013;30(8):1024-31.

25. Raff H, Ettema SL, Eastwood DC, Woodson BT. Salivary cortisol in obstructive sleep apnea: the effect of CPAP. Endocrine. 2011;137-9.
26. Panaree B, Chantana M, Wasana S, Chairat N. Effects of obstructive sleep apnea on serum brain-derived neurotrophic factor protein, cortisol, and lipid levels. Sleep Breath. 2011;649-56. https://doi.org/10.1007/s 11325-010-0415-7

27. Schmoller A, Eberhardt F, JauchChara K, Schweiger U, Zabel P, Peters A, et al. Continuous positive airway pressure therapy decreases evening cortisol concentrations in patients with severe obstructive sleep apnea. Metab Clin Exp. 2009;58(6):848-53. http://dx.doi.org/1 0.1016/j.metabol.2009.02.014

28. Romero Enrique C. Estrés y cortisol: implicaciones en la ingesta de alimento. Rev Cubana Investig Biomed [internet]. 2018;37(3):1-15.

Disponible en: https://www.medigraphi c.com/cgi-bin/new/resumen.cgi?IDAR TICULO $=88969$

29. García M del PM. Ghrelina: más allá de la regulación del hambre. Rev Gastroenterol Mex. 2005;70.

30. Geisel O, Hellweg R, Wernecke K, Wiedemann K, Müller CA. Total and acylated ghrelin plasma levels as potential longterm response markers in alcoholdependent patients receiving highdose of the GABA-B receptor agonist baclofen. Psychiatry Res. 2019;272(June 2018):431-7. https://do i.org/10.1016/j.psychres.2018.12.095

31. Farokhnia M, Grodin EN, Oot EN, Blackburn AN, Stangl BL, Schwandt ML, et al. Exogenous ghrelin administration increases alcohol selfadministration and modulates brain functional activity in heavy-drinking alcohol-dependent individuals. Nat Publ Gr. 2017 Aug:1-10. http://dx.doi. org/10.1038/mp.2017.226 
32. Santis S De, Cambi J, Tatti P, Bellussi L, Passali D. Changes in ghrelin, leptin and pro-inflammatory cytokines after therapy in Obstructive Sleep Apnea Syndrome (OSAS) patients. Otolaryngol Pol. 2015;6(2).

33. Zirlik S, Hauck T, Fuchs FS, Neurath MF, Konturek PC, Harsch IA. Leptin, obestatin and apelin levels in patients with obstructive sleep apnoea syndrome. Med Sci Monit. 2011;17(3):159-64. https://doi.org/10. 12659/MSM.881450

34. Ciftci TU, Kokturk O, Bukan N, Bilgihan A. Leptin and ghrelin levels in patients with obstructive sleep apnea. Respiration. 2005;72(4):395-401. http s://doi.org/10.1159/000086254

35. Panagopoulos VN, Ralevski E. The role of ghrelin in addiction: a review. Psychopharmacology (Berl). 2014;231(14):2725-40. https://doi.org/ 10.1007/s00213-014-3640-0

36. Harsch IA, Konturek PC, Koebnick C, Kuehnlein PP, Fuchs FS, Schahin SP, et al. Leptin and ghrelin levels in patients with obstructive sleep apnoea: effect of CPAP treatment. 2003;251-7. 37. Heilig M, Moechars D, Jerlhag E, Egecioglu E, Landgren S, Salome N, et al. Requirement of central ghrelin signaling for alcohol reward. Proc Natl Acad Sci. 2009;106(27):11318-23. htt ps://doi.org/10.1073/pnas.0812809106

38. Uribe Wiechers AC. Consecuencias metabólicas de la apnea del sueño. Neurol Neurocir y Psiquiatr. 2018;46(2):65-71.

39. Imayama I, Prasad B. Role of leptin in obstructive sleep apnea. Ann Am Thorac Soc. 2017;(20):1607-21.

40. Messenger SA, Moreau JM, Ciriello J. Effect of chronic intermittent hypoxia on leptin and leptin receptor protein expression in the carotid body.
Brain Res. 2013;1513:51-60. http://dx. doi.org/10.1016/j.brainres.2013.03.022

41. Ozturk L, U\#nal M. The association of the severity of obstructive sleep apnea with plasma leptin levels. Arch Otolaryngol Head Neck Surg. 2015;129(May 2003):538-40.

42. Lin C, Wang Y, Lee K, Liaw S, Chiu C. Effect of uvulopalatopharyngoplasty on leptin and endothelial function in sleep apnea. Ann Otol Rhinol Laryngol. 2015;123(1):40-6.

43. Pan W, Kastin AJ. Leptin: a biomarker for sleep disorders? Sleep Med Rev. 2014;18(3):283-90. http://dx .doi.org/10.1016/j.smrv.2013.07.003

44. Zhang $D$, Pang $X$, Huang R, Gong F, Zhong X, Xiao Y. Adiponectin, omentin, ghrelin, and visfatin levels in obese patients with severe obstructive sleep apnea. Biomed Res Int. 2018;2018:3410135. https://d oi.org/10.1155/2018/3410135

45. Antonopoulou S, Loukides S, Papatheodorou G, Roussos C, Alchanatis M. Airway inflammation in obstructive sleep apnea: Is leptin the missing link? Respir Med. 2008 Oct;102(10):1399-405. https://doi.org/ 10.1016/j.rmed.2008.04.021

46. Hanaoka M, Yu X, Urushihata K, Ota M, Fujimoto K, Kubo K. Leptin and leptin receptor gene polymorphisms in obstructive sleep apnea syndrome. Chest. 2008;133(1):79-85. http://dx.doi.org/1 0.1378/chest.07-1633

47. Erim A, Emre A, Yalç A. The serum leptin level in non-obese patients with obstructive sleep apnea. Auris Nasus Larynx. 2017;9-13.

48. Berger S, Polotsky V. Leptin and leptin resistance in the pathogenesis of obstructive sleep apnea: a possible link to oxidative stress and cardiovascular 
complications. Oxid Med Cell Longev. 2018;2018:5137947. https://doi.org/10 $.1155 / 2018 / 5137947$.

49. Dubey A, Kant S, Tiwari S, Agarwal S. Leptin level correlates with obesity and health related quality of life in obstructive sleep apnea syndrome patients. Indian J Tuberc. 2015;62 (2):105-9. http://dx.doi.org/10 $.1016 / j . i j t b .2015 .04 .010$

50. Yosunkaya Ş, Okur HK, Can Ü, Zamani A, Kutlu R. Impact of continuous positive airway pressure treatment on leptin levels in patients with obstructive sleep apnea syndrome. Metab Syndr Relat Disord. 2015;13(6):272-7. https://doi.org/10.1 089/met.2014.0161

51. Schiza SE, Mermigkis C. Leptin and leptin receptor gene polymorphisms and obstructive sleep apnea syndrome: is there an association? Sleep Breath. 2015;19(3):1073-8. https://doi.org/10. 1007/s11325-015-1120-3

52. Chen X, Niu X, Xiao Y, Dong J, Lu M, Kong W. Effect of continuous positive airway pressure on leptin levels in patients with obstructive sleep apnea: a meta-analysis. Otolaryngol Neck Surg. 2014;1-9.

53. Olea E, Ribeiro MJ, Yubero S, Rigual R, Masa JF, Obeso A, et al. The carotid body does not mediate the acute ventilatory effects of leptin. Arter Chemorecept Physiol Pathophysiol. 2015;379-85.

54. Espriella Guerrero RA, Rodríguez V, Rincón CJ, Cabrera D, Pérez Rodríguez SJ, Gómez-Restrepo C. Consumo de alcohol en la población colombiana: Encuesta Nacional de Salud Mental 2015. Rev Colomb Psiquiatr. 2016;5(suppl 1):76-88. http:/ /dx.doi.org/10.1016/j.rcp.2016.05.002

55. Páez-Moya S, Vega-Osorio PA. Factores de riesgo y asociados al síndrome de apneahipopnea obstructiva del sueño (SAHOS). Rev Fac Med. 2017;65 (suppl):S21-4. http://dx.doi.or g/10.15446/revfacmed.v65n1Sup.5964 6

56. Kupfer D, Regier DA. Manual diagno\#stico y estadi\#stico de los

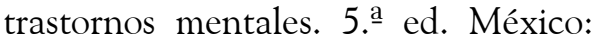
AAP; 2018. https://psychiatryonline.or $\mathrm{g}$

57. Prakash B, Foroughi M, Saeidifard F, Chakravorty S. The impact of alcohol on breathing parameters during sleep: a systematic review and metaanalysis. Sleep Med Rev. 2018;42:59-67. https:/ /doi.org/10.1016/j.smrv.2018.05.007

58. Baik I, Seo HS, Yoon D, Kim SH, Shin C. Associations of sleep apnea, NRG1 polymorphisms, alcohol consumption, and cerebral white matter hyperintensities: analysis with genome-wide association data. Sleep Apnea. 2015;38(7):1137-43. htt p://dx.doi.org/10.5665/sleep.4830

59. Simou E, Britton J, Leonardi-Bee J. Alcohol and the risk of sleep apnoea: A systematic review and meta-analysis. Sleep Med. 2018;48:38-46. https://doi. org/10.1016/j.sleep.2017.12.005

60. Nerfeldt P, Graf P, Borg S, Friberg D. Prevalence of high alcohol and benzodiazepine consumption in sleep apnea patients studied with blood and urine tests. Acta Otolaryngol. 2004;1187-91.

61. Friedmann PD. Alcohol use in adults. N Engl J Med. 2013 Apr 25;368(17):1655-6. https://doi.org/10. 1056/NEJMc1302445

62. Pilatti A, Rivarola Montejano G, Cesar A, Florencia Bartone M, Cumin G, Pautassi RM. Efecto del estrés social agudo sobre impulsividad, toma de riesgos y sesgos atencionales en jóvenes con y sin historia familiar 
de abuso de alcohol. Suma Psicol. 2017;24(2):115-28. https://doi.org/10. 1016/j.sumpsi.2016.09.003

63. Vahia VN. Diagnostic and statistical manual of mental disorders 5: A quick glance. Indian J Psychiatry. 2013;55(3):220-3. https://doi.org/10.4 103/0019-5545.117131 